J. Phys. Chem. A. 2016

\title{
Benchmark Thermodynamic Properties of Methyl- and Methoxy- Benzamides: Comprehensive Experimental and Theoretical Study
}

Vladimir N. Emel'yanenko, ${ }^{a}$ Ksenia V. Zaitseva, ${ }^{a}$ Ruslan N. Nagrimanov, ${ }^{b}$ Boris N. Solomonov, ${ }^{\mathrm{b}, *}$ Sergey P. Verevkin ${ }^{\mathrm{a}, * *}$

${ }^{a}$ Department of Physical Chemistry and Department ,, Science and Technology of Life, Light and Matter", University of Rostock, Dr-Lorenz-Weg 1, D-18059, Rostock, Germany

${ }^{b}$ Department of Physical Chemistry, Kazan Federal University, Kremlevskaya str. 18, 420008 Kazan, Russia

* To whom correspondence concerning the solution calorimetry experiments (Kazan) and correlations should be addressed, E-mail: boris.solomonov@kpfu.ru (B.N. Solomonov) ** To whom correspondence concerning the combustion, vaporization/sublimation experiments (Rostock), data evaluation and correlations should be addressed, E-mail: sergey.verevkin@unirostock.de 
Table S1. Origin, Purity, Methods of Purification and Analysis of Chemicals Used in This Work

\begin{tabular}{lccccc}
\hline \multicolumn{1}{c}{ Compound } & CAS & Source & Initial purity & Purification method & Final purity $^{a}$ \\
\hline 2- $\mathrm{CH}_{3}$-benzamide (cr) & $527-85-3$ & Aldrich & 0.99 & sublimation & 0.999 \\
3- $\mathrm{CH}_{3}$-benzamide (cr) & $618-47-3$ & Alfa & 0.99 & sublimation & 0.999 \\
4- $\mathrm{CH}_{3}$-benzamide (cr) & $619-55-6$ & Aldrich & 0.99 & sublimation & 0.999 \\
2- $\mathrm{CH}_{3} \mathrm{O}$-benzamide (cr) & $2439-77-2$ & ABCR & 0.98 & sublimation & 0.999 \\
3- $\mathrm{CH}_{3} \mathrm{O}$-benzamide (cr) & $5813-86-5$ & Aldrich & 0.97 & sublimation & 0.999 \\
4- $\mathrm{CH}_{3} \mathrm{O}$-benzamide (cr) & $3424-93-9$ & Aldrich & 0.98 & sublimation & 0.999 \\
acetonitrile (l) & $75-05-8$ & Ekos-1 & 0.990 & distillation & $0.999^{b}$ \\
potassium chloride (cr) & $7447-40-7$ & Aldrich & 0.999 & none & -
\end{tabular}

${ }^{a}$ Gas chromatography. ${ }^{b}$ Mass fraction of water 0.0004 according to the Karl Fisher titration.

Table S2. Results from Transpiration Method: Absolute Vapor Pressures $p$, Standard Molar Sublimation Enthalpies and Standard Molar Sublimation Entropies

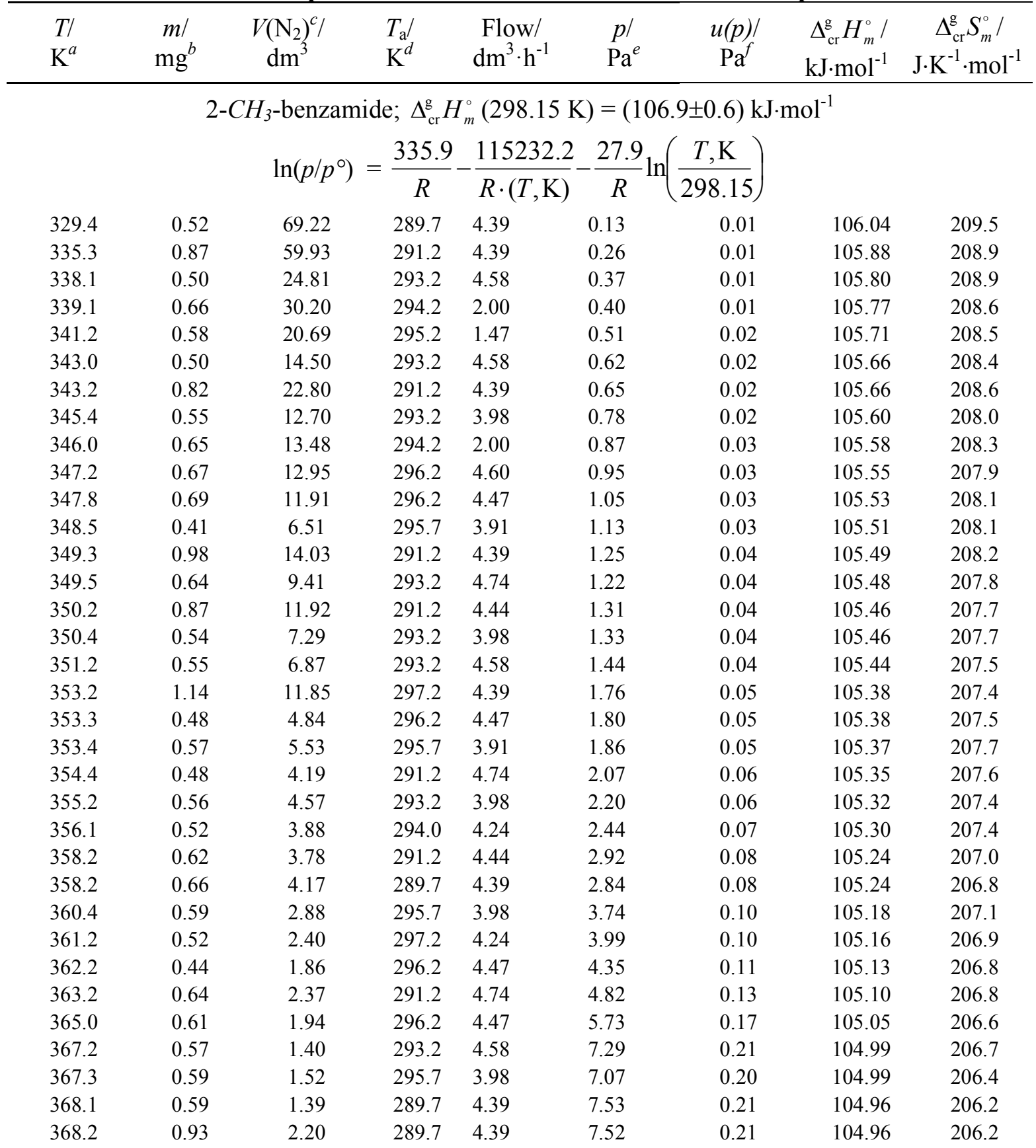




$\begin{array}{lllllllll}368.3 & 0.65 & 1.49 & 296.2 & 4.47 & 7.90 & 0.22 & 104.96 & 206.5 \\ 369.3 & 0.53 & 1.11 & 295.7 & 3.91 & 8.69 & 0.24 & 104.93 & 206.4 \\ 370.2 & 0.71 & 1.37 & 293.2 & 4.58 & 9.37 & 0.26 & 104.91 & 206.3 \\ 371.2 & 0.75 & 1.38 & 297.2 & 4.39 & 10.00 & 0.28 & 104.88 & 206.0 \\ 371.3 & 0.60 & 1.04 & 291.2 & 4.44 & 10.33 & 0.28 & 104.87 & 206.2\end{array}$

3- $\mathrm{CH}_{3}$-benzamide; $\Delta_{\mathrm{cr}}^{\mathrm{g}} H_{m}^{\circ}(298.15 \mathrm{~K})=(106.6 \pm 0.7) \mathrm{kJ} \cdot \mathrm{mol}^{-1}$ $\ln \left(p / p^{\circ}\right)=\frac{336.0}{R}-\frac{114914.1}{R \cdot(T, \mathrm{~K})}-\frac{27.9}{R} \ln \left(\frac{T, \mathrm{~K}}{298.15}\right)$

\begin{tabular}{|c|c|c|c|c|c|c|c|c|}
\hline 329.7 & 0.42 & 48.62 & 297.2 & 4.27 & 0.16 & 0.01 & 105.71 & 209.6 \\
\hline 336.9 & 0.42 & 21.25 & 298.4 & 4.25 & 0.37 & 0.01 & 105.51 & 209.1 \\
\hline 338.1 & 0.56 & 24.81 & 294.2 & 4.58 & 0.41 & 0.02 & 105.48 & 208.9 \\
\hline 339.1 & 0.76 & 30.03 & 291.2 & 2.00 & 0.45 & 0.02 & 105.45 & 208.7 \\
\hline 341.2 & 0.64 & 20.69 & 294.2 & 1.47 & 0.56 & 0.02 & 105.40 & 208.4 \\
\hline 342.4 & 0.54 & 15.31 & 294.7 & 4.59 & 0.64 & 0.02 & 105.36 & 208.3 \\
\hline 343.0 & 0.55 & 14.50 & 293.2 & 4.58 & 0.69 & 0.02 & 105.35 & 208.3 \\
\hline 343.9 & 0.46 & 10.96 & 298.4 & 4.53 & 0.77 & 0.02 & 105.32 & 208.3 \\
\hline 345.4 & 0.61 & 12.70 & 295.7 & 3.98 & 0.88 & 0.03 & 105.28 & 208.0 \\
\hline 346.0 & 0.72 & 13.45 & 291.2 & 2.00 & 0.95 & 0.03 & 105.26 & 208.1 \\
\hline 346.3 & 0.49 & 9.09 & 298.0 & 4.36 & 0.98 & 0.03 & 105.25 & 208.1 \\
\hline 347.2 & 0.77 & 13.10 & 293.2 & 4.60 & 1.06 & 0.03 & 105.23 & 207.9 \\
\hline 348.5 & 0.45 & 6.51 & 292.2 & 3.91 & 1.25 & 0.04 & 105.19 & 208.0 \\
\hline 349.5 & 0.70 & 8.93 & 293.2 & 4.74 & 1.42 & 0.04 & 105.16 & 208.1 \\
\hline 350.4 & 0.60 & 7.29 & 295.7 & 3.98 & 1.49 & 0.04 & 105.14 & 207.7 \\
\hline 351.0 & 0.51 & 5.65 & 298.4 & 4.52 & 1.66 & 0.05 & 105.12 & 208.0 \\
\hline 351.2 & 0.61 & 6.56 & 293.2 & 4.58 & 1.67 & 0.05 & 105.12 & 207.9 \\
\hline 353.2 & 1.26 & 11.34 & 294.0 & 4.39 & 2.00 & 0.06 & 105.06 & 207.6 \\
\hline 353.4 & 0.63 & 5.53 & 295.7 & 3.91 & 2.06 & 0.06 & 105.06 & 207.6 \\
\hline 353.7 & 0.49 & 4.36 & 295.0 & 4.36 & 2.05 & 0.06 & 105.05 & 207.3 \\
\hline 354.4 & 0.54 & 4.19 & 293.2 & 4.74 & 2.31 & 0.06 & 105.03 & 207.6 \\
\hline 355.2 & 0.62 & 4.57 & 295.7 & 3.98 & 2.46 & 0.07 & 105.01 & 207.4 \\
\hline 356.1 & 0.58 & 3.74 & 293.2 & 4.24 & 2.80 & 0.08 & 104.98 & 207.7 \\
\hline 356.3 & 0.42 & 2.70 & 296.2 & 4.15 & 2.81 & 0.08 & 104.97 & 207.5 \\
\hline 357.8 & 0.56 & 3.23 & 295.5 & 4.50 & 3.17 & 0.08 & 104.93 & 207.2 \\
\hline 360.0 & 0.70 & 3.23 & 297.4 & 4.13 & 3.96 & 0.10 & 104.87 & 207.1 \\
\hline 360.4 & 0.66 & 2.88 & 295.7 & 3.98 & 4.14 & 0.11 & 104.86 & 207.1 \\
\hline 361.2 & 0.58 & 2.33 & 295.2 & 4.24 & 4.53 & 0.12 & 104.84 & 207.1 \\
\hline 362.5 & 0.58 & 2.04 & 296.2 & 4.36 & 5.18 & 0.13 & 104.80 & 207.1 \\
\hline 363.2 & 0.71 & 2.37 & 293.2 & 4.74 & 5.37 & 0.16 & 104.78 & 206.8 \\
\hline 364.8 & 0.56 & 1.64 & 296.7 & 4.36 & 6.19 & 0.18 & 104.74 & 206.5 \\
\hline 367.0 & 0.52 & 1.22 & 296.2 & 3.84 & 7.73 & 0.20 & 104.68 & 206.5 \\
\hline 367.2 & 0.63 & 1.47 & 298.2 & 4.42 & 7.87 & 0.22 & 104.67 & 206.5 \\
\hline 367.2 & 0.63 & 1.40 & 293.2 & 4.58 & 8.08 & 0.23 & 104.67 & 206.7 \\
\hline 367.3 & 0.66 & 1.52 & 295.7 & 3.98 & 7.83 & 0.22 & 104.67 & 206.4 \\
\hline 368.1 & 0.65 & 1.39 & 297.2 & 4.39 & 8.56 & 0.24 & 104.65 & 206.5 \\
\hline 369.3 & 0.59 & 1.11 & 295.7 & 3.91 & 9.65 & 0.27 & 104.61 & 206.4 \\
\hline 370.2 & 0.79 & 1.37 & 293.2 & 4.58 & 10.36 & 0.28 & 104.59 & 206.3 \\
\hline 371.2 & 0.83 & 1.38 & 296.2 & 4.39 & 11.02 & 0.30 & 104.56 & 205.9 \\
\hline
\end{tabular}

4- $\mathrm{CH}_{3}$-benzamide; $\Delta_{\mathrm{cr}}^{\mathrm{g}} H_{m}^{\circ}(298.15 \mathrm{~K})=(108.9 \pm 0.9) \mathrm{kJ} \cdot \mathrm{mol}^{-1}$ $\ln \left(p / p^{\circ}\right)=\frac{331.2}{R}-\frac{117165.7}{R \cdot(T, \mathrm{~K})}-\frac{27.9}{R} \ln \left(\frac{T, \mathrm{~K}}{298.15}\right)$

$\begin{array}{lllllllll}343.9 & 0.59 & 54.34 & 296.2 & 4.53 & 0.20 & 0.01 & 107.57 & 203.6 \\ 346.6 & 0.72 & 50.19 & 298.0 & 4.36 & 0.26 & 0.01 & 107.49 & 203.3 \\ 350.4 & 0.52 & 24.00 & 301.1 & 4.68 & 0.40 & 0.02 & 107.39 & 203.1 \\ 352.7 & 0.49 & 17.58 & 294.7 & 4.63 & 0.51 & 0.02 & 107.33 & 203.0\end{array}$




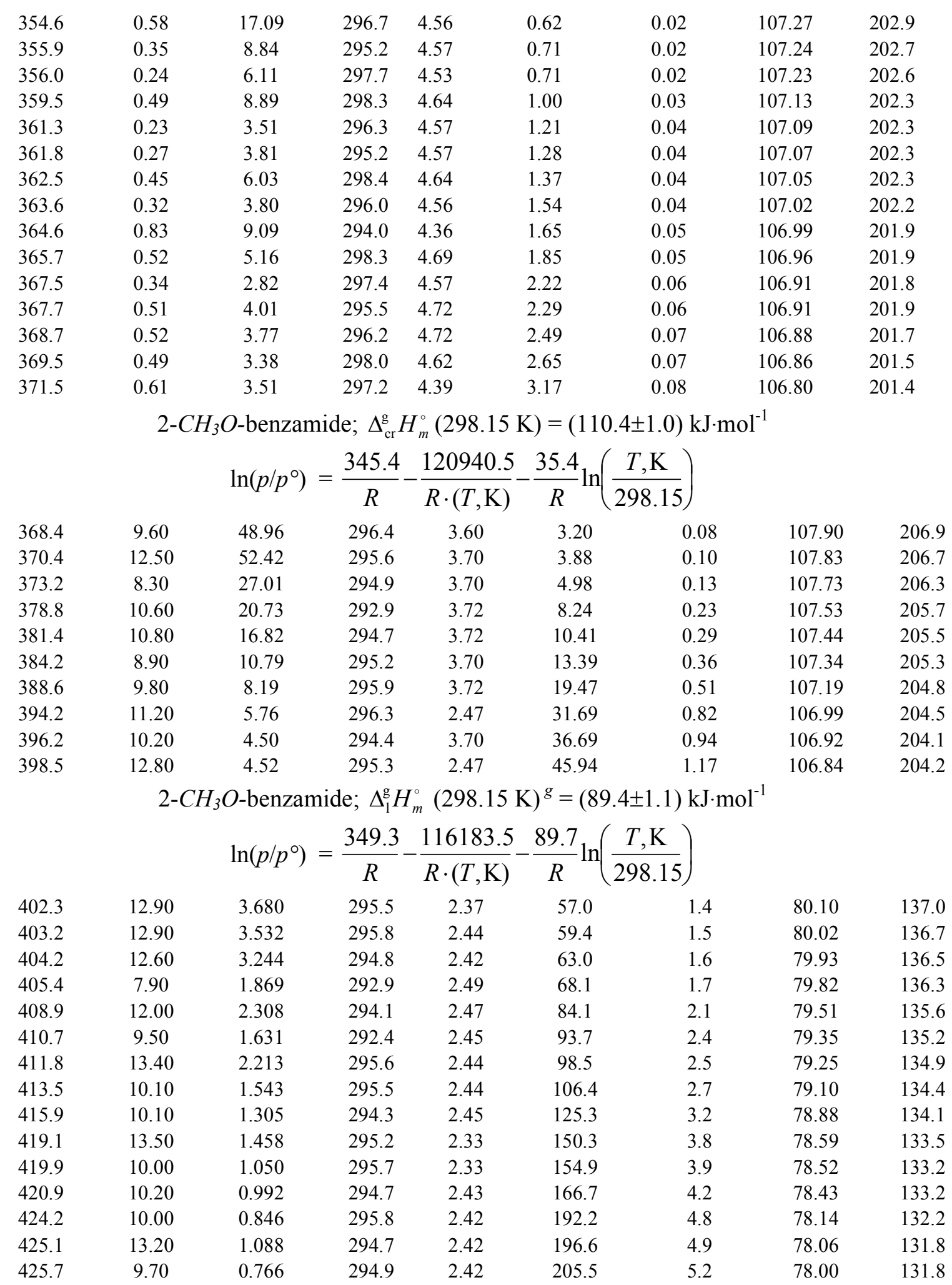

3- $\mathrm{CH}_{3} \mathrm{O}$-benzamide; $\Delta_{\mathrm{cr}}^{\mathrm{g}} H_{m}^{\circ}(298.15 \mathrm{~K})=(120.6 \pm 1.1) \mathrm{kJ} \cdot \mathrm{mol}^{-1}$ $\ln \left(p / p^{\circ}\right)=\frac{363.2}{R}-\frac{131192.9}{R \cdot(T, \mathrm{~K})}-\frac{35.4}{R} \ln \left(\frac{T, \mathrm{~K}}{298.15}\right)$

$\begin{array}{lcccccccc}372.3 & 5.30 & 61.02 & 295.7 & 3.24 & 1.41 & 0.04 & 118.02 & 224.2 \\ 375.7 & 8.30 & 66.70 & 295.7 & 3.14 & 2.02 & 0.06 & 117.89 & 224.0 \\ 379.2 & 12.00 & 68.46 & 295.2 & 3.27 & 2.85 & 0.08 & 117.77 & 223.6 \\ 383.7 & 10.90 & 40.69 & 291.2 & 3.27 & 4.29 & 0.11 & 117.61 & 222.9 \\ 386.2 & 10.00 & 29.85 & 295.7 & 3.12 & 5.45 & 0.14 & 117.52 & 222.7\end{array}$




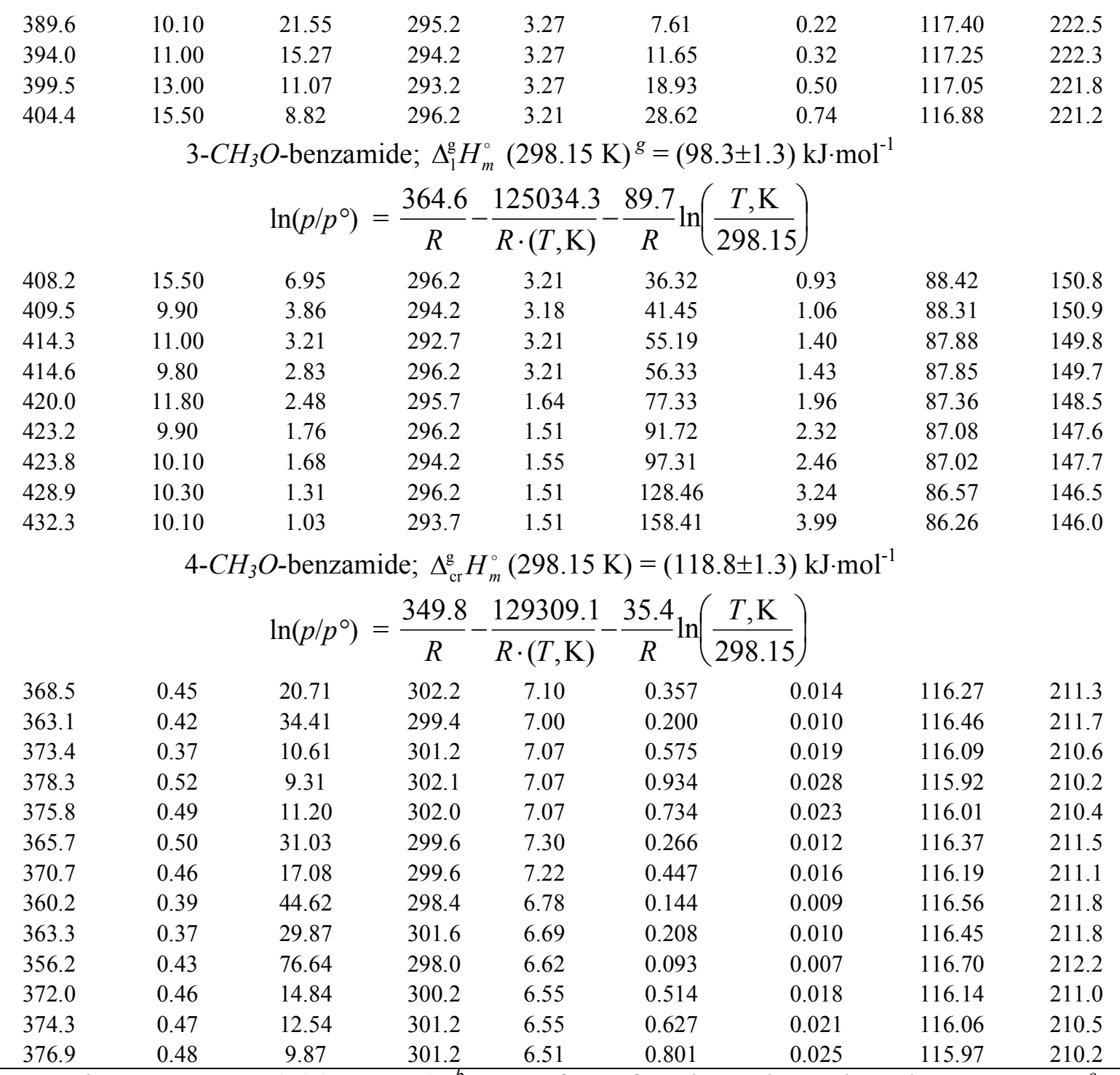

${ }^{a}$ Saturation temperature $(u(T)=0.1 \mathrm{~K}) .{ }^{b}$ Mass of transferred sample condensed at $T=243 \mathrm{~K} .{ }^{c}$ Volume of nitrogen $\left(u(V)=0.005 \mathrm{dm}^{3}\right)$ used to transfer $m(u(m)=0.0001 \mathrm{~g})$ of the sample. ${ }^{d} T_{\mathrm{a}}$ is the temperature of the soap bubble meter used for measurement of the gas flow. ${ }^{e}$ Vapor pressure at temperature $T$, calculated from the $m$ and the residual vapor pressure at the condensation temperature calculated by an iteration procedure. ${ }^{f}$ Uncertainties were calculated with $u(p / \mathrm{Pa})=0.005+0.025(p / \mathrm{Pa})$ for pressures below $5 \mathrm{~Pa}$ and with $u(p / \mathrm{Pa})=0.025+$ $0.025(\mathrm{p} / \mathrm{Pa})$ for pressure from 5 to $3000 \mathrm{~Pa} .{ }^{g}$ Measured over the liquid sample. 
Table S3. Experimental Enthalpies of Solution of Substituted Benzamides in Acetonitrile Measured in this Work at 298.15 $\mathrm{K}$ and $0.1 \mathrm{MPa}^{a}$

\begin{tabular}{|c|c|c|c|c|}
\hline Solute & Solvent & Mass of sample $\mathrm{e}^{b} / \mathrm{mg}$ & $\begin{array}{l}\text { Molality }{ }^{c} / \\
\mathrm{mmol} \cdot \mathrm{kg}^{-1}\end{array}$ & $\begin{array}{c}\Delta_{\text {soln }} H_{m}^{A i / S d} / \\
\mathrm{kJ} \mathrm{mol}^{-1}\end{array}$ \\
\hline \multirow[t]{5}{*}{ potassium chloride } & water & 207.38 & 27.82 & 17.46 \\
\hline & & 206.93 & 27.76 & 17.40 \\
\hline & & 206.73 & 27.73 & 17.37 \\
\hline & & 208.06 & 27.91 & 17.41 \\
\hline & & & \multicolumn{2}{|c|}{$\Delta_{\text {soln }} H_{m}^{A i / S}=17.41 \pm 0.04 \mathrm{~kJ} \mathrm{~mol}^{-1}$} \\
\hline \multirow[t]{5}{*}{ 3- $\mathrm{CH}_{3}$-benzamide } & acetonitrile & 25.00 & 2.38 & 24.61 \\
\hline & & 24.97 & 2.38 & 24.39 \\
\hline & & 21.80 & 4.46 & 24.62 \\
\hline & & 27.72 & 5.02 & 24.85 \\
\hline & & & \multicolumn{2}{|c|}{$\Delta_{\mathrm{soln}} H_{m}^{A i / S}=24.6 \pm 0.2 \mathrm{~kJ} \cdot \mathrm{mol}^{-1 e}$} \\
\hline \multirow[t]{5}{*}{ 4- $\mathrm{CH}_{3}$-benzamide } & acetonitrile & 19.46 & 1.85 & 25.29 \\
\hline & & 19.33 & 1.84 & 25.62 \\
\hline & & 15.88 & 3.37 & 25.75 \\
\hline & & 16.60 & 3.42 & 26.44 \\
\hline & & & \multicolumn{2}{|c|}{$\Delta_{\text {soln }} H_{m}^{A i / S}=25.8 \pm 0.5 \mathrm{~kJ} \cdot \mathrm{mol}^{-1}$} \\
\hline \multirow[t]{5}{*}{ 4- $\mathrm{CH}_{3} \mathrm{O}$-benzamide } & acetonitrile & 26.63 & 2.27 & 26.53 \\
\hline & & 44.70 & 3.81 & 26.11 \\
\hline & & 34.85 & 5.23 & 26.63 \\
\hline & & 63.84 & 9.24 & 25.98 \\
\hline & & & \multicolumn{2}{|c|}{$\Delta_{\text {soln }} H_{m}^{A / S}=26.3 \pm 0.3 \mathrm{~kJ} \cdot \mathrm{mol}^{-1}$} \\
\hline
\end{tabular}

${ }^{a}$ Standard uncertainties $\mathrm{u}$ are $u(T)=0.01 \mathrm{~K}, \mathrm{u}(\mathrm{p})=5 \mathrm{kPa} .{ }^{b}$ Mass of solute sample which was added in each dissolution experiment. ${ }^{c}$ Molality of solute in solution after experiments. Standard uncertainties $u$ are $u(\mathrm{~b})=0.08 \mathrm{mmol} \cdot \mathrm{kg}^{-1} .{ }^{d}$ Enthalpy of solution of each experiment. ${ }^{\mathrm{e}}$ Average enthalpy of solution. Uncertainties correspond to expanded uncertainties of the mean $U(0.95$ level of confidence, $\mathrm{k}=2$ ).

Table S4. Formula, Density $\rho(T=293 \mathrm{~K})$, Specific Heat Capacity $c_{p}(T=298 \mathrm{~K})$, and Expansion Coefficients $(\delta V / \delta T)_{p}$ of the Materials Used in the Present Study

\begin{tabular}{|c|c|c|c|c|}
\hline Compounds & Formula & $\frac{\rho}{\mathrm{g} \cdot \mathrm{cm}^{-3}}$ & $\frac{c_{p}{ }^{a}}{\mathrm{~J} \cdot \mathrm{K}^{-1} \mathrm{o}^{-1}}$ & $\frac{10^{-6} \cdot(\delta V / \delta T)_{\mathrm{p}}}{\mathrm{dm}^{3} \cdot \mathrm{K}^{-1}}$ \\
\hline 2- $\mathrm{CH}_{3}$-benzamide & $\mathrm{C}_{8} \mathrm{H}_{9} \mathrm{O}_{1} \mathrm{~N}_{1}$ & $1.16^{1}$ & $\begin{array}{c}8 \\
1.34\end{array}$ & 0.1 \\
\hline 3- $\mathrm{CH}_{3}$-benzamide & $\mathrm{C}_{8} \mathrm{H}_{9} \mathrm{O}_{1} \mathrm{~N}_{1}$ & $1.19^{1}$ & 1.34 & 0.1 \\
\hline 4- $\mathrm{CH}_{3}$-benzamide & $\mathrm{C}_{8} \mathrm{H}_{9} \mathrm{O}_{1} \mathrm{~N}_{1}$ & $1.18^{2}$ & 1.34 & 0.1 \\
\hline 2- $\mathrm{CH}_{3} \mathrm{O}$-benzamide & $\mathrm{C}_{8} \mathrm{H}_{9} \mathrm{O}_{2} \mathrm{~N}_{1}$ & $1.14^{2}$ & 1.53 & 0.1 \\
\hline $\begin{array}{l}\text { polyethylene } \\
\text { cotton }^{\mathrm{c}}\end{array}$ & $\begin{array}{c}\mathrm{CH}_{1.93} \\
\mathrm{CH}_{1.774} \mathrm{O}_{0.887}\end{array}$ & $\begin{array}{l}0.92 \\
1.50\end{array}$ & $\begin{array}{l}2.53 \\
1.67\end{array}$ & $\begin{array}{l}0.1 \\
0.1\end{array}$ \\
\hline
\end{tabular}

${ }^{a}$ Estimated according to ref. ${ }^{3}{ }^{b}$ Energy of combustion $\Delta_{\mathrm{c}} u^{\circ}$ (polyethylene) $=-46357.3 \mathrm{~J} \cdot \mathrm{g}^{-1}$; $u\left(\Delta_{\mathrm{c}} u^{\circ}\right)=3.6 \mathrm{~J} \cdot \mathrm{g}^{-1} \cdot{ }^{c}$ Energy of combustion $\Delta_{\mathrm{c}} u^{\circ}($ cotton $)=-16945.2 \mathrm{~J} \cdot \mathrm{g}^{-1} ; u\left(\Delta_{\mathrm{c}} u^{\circ}\right)=4.2 \mathrm{~J} \cdot \mathrm{g}^{-1}$. 
Table S5. Results for Combustion Experiments at $T=298.15 \mathrm{~K}\left(\mathrm{p}^{\circ}=0.1 \mathrm{MPa}\right)$ for the 2$\mathrm{CH}_{3}$-benzamide ${ }^{a}$

\begin{tabular}{lccccc}
\hline $\mathrm{m}$ (substance) $/ \mathrm{g}$ & 0.260459 & 0.520114 & 0.353425 & 0.259643 & 0.242586 \\
$\mathrm{~m}^{\prime}($ cotton $) / \mathrm{g}$ & 0.001058 & 0.001185 & 0.00092 & 0.000858 & 0.001167 \\
$\mathrm{~m}^{\prime \prime}($ polyethene) $/ \mathrm{g}$ & 0.376728 & 0.206469 & 0.303945 & 0.383047 & 0.404673 \\
$\Delta \mathrm{T}_{\mathrm{c}} / \mathrm{K}^{\mathrm{c}}$ & 1.71945 & 1.73062 & 1.68607 & 1.73701 & 1.76834 \\
$\left(\varepsilon_{\text {calor }}\right) \cdot\left(-\Delta \mathrm{T}_{\mathrm{c}}\right) / \mathrm{J}$ & -25601 & -25767.4 & -25104.1 & -25862.6 & -26329 \\
$\left(\varepsilon_{\text {cont }}\right) \cdot\left(-\Delta \mathrm{T}_{\mathrm{c}}\right) / \mathrm{J}$ & -27.84 & -27.87 & -27.41 & -28.2 & -28.63 \\
$\Delta \mathrm{U}_{\text {decomp }} \mathrm{HNO}_{3} / \mathrm{J}$ & 51.36 & 59.13 & 53.16 & 53.75 & 53.75 \\
$\Delta \mathrm{U}_{\text {corr }} / \mathrm{Jd}^{\mathrm{d}}$ & 10.03 & 12.5 & 10.72 & 10.09 & 10.09 \\
$-\mathrm{m}^{\prime} \cdot \Delta_{\mathrm{c}} \mathrm{u}^{\prime} / \mathrm{J}$ & 17.93 & 20.08 & 15.59 & 14.54 & 19.77 \\
$-\mathrm{m}^{\prime \prime} \cdot \Delta_{\mathrm{c}} \mathrm{u}^{\prime \prime} / \mathrm{J}$ & 17464.09 & 9571.35 & 14090.07 & 17757.02 & 18759.55 \\
$\Delta_{\mathrm{c}} \mathrm{u}^{\circ}(\mathrm{cr}) /\left(\mathrm{J} \cdot \mathrm{g}^{-1}\right)$ & -31043.1 & -31016.7 & -31016.5 & -31024.7 & -30976.3 \\
\hline$-\Delta_{\mathrm{c}} \mathrm{u}^{\circ}(\mathrm{cr}) /\left(\mathrm{J} \cdot \mathrm{g}^{-1}\right)$ & \multicolumn{5}{c}{$31015.5 \pm 10.9$} \\
\hline \multicolumn{5}{c}{$-4195.3 \pm 3.1$} \\
\hline$\Delta_{\mathrm{c}} H_{\mathrm{m}}^{\circ}(\mathrm{cr})$ & \multicolumn{5}{c}{} \\
\hline
\end{tabular}

${ }^{\text {a }}$ The definition of the symbols assigned according to ref. [4] is as follows: $m$ (substance), $m^{\prime}$ (cotton) and $m$ "(polythene) are, respectively, the mass of compound burnt, the mass of fuse (cotton), and the mass of polyethylene bag used in each experiment, masses were corrected for buoyancy; $V($ bomb $)=0.32 \mathrm{dm}^{3}$ is the internal volume of the calorimetric bomb; $p^{\mathrm{i}}(\mathrm{gas})=3.00 \mathrm{MPa}$ is the initial oxygen pressure in the bomb; $m^{\mathrm{i}}\left(\mathrm{H}_{2} \mathrm{O}\right)=1.00 \mathrm{~g}$ is the mass of water added to the bomb for dissolution of combustion gases; $\varepsilon_{\text {calor }}=(14889.04 \pm 0.9) \mathrm{J} \cdot \mathrm{K}^{-1}$ is the energy equivalent of the calorimeter; $\Delta T_{\mathrm{c}}=T^{\mathrm{f}}-T^{\mathrm{i}}+\Delta T_{\mathrm{corr}}$ is the corrected temperature rise from initial temperature $T^{\mathrm{i}}$ to final temperature $T^{\mathrm{f}}$, with the correction $\Delta T_{\mathrm{corr}}$ for heat exchange during the experiment; $\varepsilon_{\mathrm{cont}}$ is the energy equivalents of the bomb contents in their initial $\varepsilon_{\text {cont }}^{\mathrm{i}}$ and final states $\varepsilon_{\text {cont }}^{\mathrm{f}}$, the contribution for the bomb content is calculated with $\left(\varepsilon_{\text {cont }}\right) \cdot\left(-\Delta T_{\mathrm{c}}\right)=\left(\varepsilon_{\text {cont }}^{\mathrm{i}}\right) \cdot\left(T^{\mathrm{i}}-298.15\right)+\left(\varepsilon_{\text {cont }}^{\mathrm{f}}\right) \cdot\left(298.15-T^{\mathrm{f}}+\Delta T_{\text {corr. }}\right) \cdot \Delta U_{\text {decomp }}$ $\mathrm{HNO}_{3}$ is the energy correction for the nitric acid formation. $\Delta U_{\text {corr }}$ is the correction to standard states. Uncertainties of the combustion energy are expressed as the standard deviation of the mean.

Table S6. Results for Combustion Experiments at $T=298.15 \mathrm{~K}\left(\mathrm{p}^{\circ}=0.1 \mathrm{MPa}\right)$ for the 3$\mathrm{CH}_{3}$-benzamide ${ }^{a}$

\begin{tabular}{lccccc}
\hline $\mathrm{m}$ (substance) $/ \mathrm{g}$ & 0.36076 & 0.375622 & 0.312381 & 0.179985 & 0.275406 \\
$\mathrm{~m}^{\prime}($ cotton) $/ \mathrm{g}$ & 0.001086 & 0.00121 & 0.001114 & 0.001222 & 0.00122 \\
$\mathrm{~m}^{\prime \prime}($ polyethene) $/ \mathrm{g}$ & 0.302264 & 0.307792 & 0.380991 & 0.457513 & 0.327145 \\
$\Delta \mathrm{T}_{\mathrm{c}} / \mathrm{K}^{\mathrm{c}}$ & 1.69617 & 1.74437 & 1.84028 & 1.80323 & 1.59612 \\
$\left(\varepsilon_{\text {calor }}\right) \cdot\left(-\Delta \mathrm{T}_{\mathrm{c}}\right) / \mathrm{J}$ & -25254.4 & -25972.2 & -27400.2 & -26848.5 & -23764.8 \\
$\left(\varepsilon_{\text {cont }}\right) \cdot\left(-\Delta \mathrm{T}_{\mathrm{c}}\right) / \mathrm{J}$ & -27.18 & -28.36 & -30.25 & -29.56 & -25.68 \\
$\Delta \mathrm{U}_{\text {decomp }} \mathrm{HNO}_{3} / \mathrm{J}$ & 54.35 & 55.55 & 57.34 & 52.56 & 49.57 \\
$\Delta \mathrm{U}_{\text {corr }} / \mathrm{J}^{\mathrm{d}}$ & 10.8 & 11.25 & 11.18 & 9.73 & 9.53 \\
$-\mathrm{m}^{\prime} \cdot \Delta_{\mathrm{c}} \mathrm{u}^{\prime} / \mathrm{J}$ & 18.4 & 20.5 & 18.88 & 20.71 & 20.67 \\
$-\mathrm{m}^{\prime \prime} \cdot \Delta_{\mathrm{c}} \mathrm{u}^{\prime \prime} / \mathrm{J}$ & 14012.14 & 14268.41 & 17661.71 & 21209.07 & 15165.56 \\
$\Delta_{\mathrm{c}} \mathrm{u}^{\circ}(\mathrm{cr}) /\left(\mathrm{J} \cdot \mathrm{g}^{-1}\right)$ & -31006.5 & -31001.5 & -30992.1 & -31036.1 & -31027.3 \\
\hline$-\Delta_{\mathrm{c}} \mathrm{u}^{\circ}(\mathrm{cr}) /\left(\mathrm{J} \cdot \mathrm{g}^{-1}\right)$ & \multicolumn{5}{c}{$-41012.7 \pm 8.2^{b}$} \\
\hline \multicolumn{7}{c}{$\Delta_{\mathrm{c}} H_{\mathrm{m}}^{\circ}(\mathrm{cr})$} & \multicolumn{7}{c}{$(1484.9 \pm 2.4$} \\
\hline
\end{tabular}

${ }^{a}$ The energy equivalent of the calorimeter: $\varepsilon_{\text {calor }}=(14889.04 \pm 0.9) \mathrm{J} \cdot \mathrm{K}^{-1} \cdot{ }^{b}$ Uncertainties of the combustion energy are expressed as the standard deviation of the mean. 
Table S7. Results for Combustion Experiments at $T=298.15 \mathrm{~K}\left(\mathrm{p}^{\circ}=0.1 \mathrm{MPa}\right)$ for the 4$\mathrm{CH}_{3}$-benzamide ${ }^{a}$

\begin{tabular}{lccccc}
\hline $\mathrm{m}$ (substance) $/ \mathrm{g}$ & 0.471929 & 0.487832 & 0.447509 & 0.866024 & 0.390324 \\
$\mathrm{~m}^{\prime}($ cotton $) / \mathrm{g}$ & 0.001217 & 0.000995 & 0.001209 & 0.001077 & 0.001139 \\
$\mathrm{~m}^{\prime \prime}($ polyethene) $/ \mathrm{g}$ & 0.257995 & 0.290696 & 0.267722 & 0.149281 & 0.365637 \\
$\Delta \mathrm{T}_{\mathrm{c}} / \mathrm{K}^{\mathrm{c}}$ & 1.78798 & 1.92387 & 1.76814 & 2.26979 & 1.95367 \\
$\left(\varepsilon_{\text {calor }}\right) \cdot\left(-\Delta \mathrm{T}_{\mathrm{c}}\right) / \mathrm{J}$ & -26621.4 & -28644.7 & -26326 & -33795.2 & -29088.4 \\
$\left(\varepsilon_{\text {cont }}\right) \cdot\left(-\Delta \mathrm{T}_{\mathrm{c}}\right) / \mathrm{J}$ & -28.96 & -31.7 & -28.67 & -38.19 & -32.3 \\
$\Delta \mathrm{U}_{\text {decomp }} \mathrm{HNO}_{3} / \mathrm{J}$ & 60.32 & 64.5 & 58.53 & 80.63 & 62.12 \\
$\Delta \mathrm{U}_{\text {corr }} / \mathrm{J}$ & 12.36 & 13.28 & 12.04 & 18.94 & 12.53 \\
$-\mathrm{m}^{\prime} \cdot \Delta_{\mathrm{c}} \mathrm{u}^{\prime} / \mathrm{J}$ & 20.62 & 16.86 & 20.49 & 18.25 & 19.3 \\
$-\mathrm{m}^{\prime \prime} \cdot \Delta_{\mathrm{c}} \mathrm{u}^{\prime \prime} / \mathrm{J}$ & 11959.95 & 13475.88 & 12410.87 & 6920.26 & 16949.94 \\
$\Delta_{\mathrm{c}} \mathrm{u}^{\circ}(\mathrm{cr}) /\left(\mathrm{J} \cdot \mathrm{g}^{-1}\right)$ & -30930.7 & -30965.4 & -30955.3 & -30940.6 & -30940.5 \\
\hline$-\Delta_{\mathrm{c}} \mathrm{u}^{\circ}(\mathrm{cr}) /\left(\mathrm{J} \cdot \mathrm{g}^{-1}\right)$ & \multicolumn{5}{c}{$30946.5 \pm 6.1^{b}$} \\
\hline$\Delta_{\mathrm{c}} H_{\mathrm{m}}^{\circ}(\mathrm{cr})$ & $-4185.9 \pm 1.9$ \\
\hline
\end{tabular}

${ }^{a}$ The energy equivalent of the calorimeter: $\varepsilon_{\text {calor }}=(14889.04 \pm 0.9) \mathrm{J} \cdot \mathrm{K}^{-1} \cdot{ }^{b}$ Uncertainties of the combustion energy are expressed as the standard deviation of the mean.

Table S8. Results for Combustion Experiments at $T=298.15 \mathrm{~K}\left(\mathrm{p}^{\circ}=0.1 \mathrm{MPa}\right)$ for the 2$\mathrm{CH}_{3} \mathrm{O}$-benzamide ${ }^{a}$

\begin{tabular}{lcccc}
\hline$m$ (substance) $/ \mathrm{g}$ & 0.483853 & 0.557408 & 0.735341 & 0.383516 \\
$m^{\prime}$ (cotton) $/ \mathrm{g}$ & 0.000882 & 0.001028 & 0.000961 & 0.000853 \\
$m^{\prime \prime}($ polyethylene) $/ \mathrm{g}$ & 0.229666 & 0.224405 & 0.204114 & 0.217922 \\
$\Delta T_{\mathrm{c}} / \mathrm{K}$ & 1.60027 & 1.71795 & 1.97732 & 1.37999 \\
$\left(\varepsilon_{\text {calor }}\right) \cdot\left(-\Delta \mathrm{T}_{\mathrm{c}}\right) / \mathrm{J}$ & -23710 & -25453.7 & -29296.6 & -20446.4 \\
$\left(\varepsilon_{\text {cont }}\right) \cdot\left(-\Delta \mathrm{T}_{\mathrm{c}}\right) / \mathrm{J}$ & -25.53 & -27.68 & -32.63 & -21.58 \\
$\Delta U_{\text {decomp }} \mathrm{HNO}_{3} / \mathrm{J}$ & 51.36 & 55.84 & 65.10 & 37.63 \\
$\Delta U_{\text {corr }} / \mathrm{J}$ & 11.71 & 13.07 & 16.34 & 9.64 \\
$-m^{\prime} \cdot \Delta_{\mathrm{c}} u^{\prime} / \mathrm{J}$ & 14.95 & 17.42 & 16.28 & 14.45 \\
$-m^{\prime \prime} \cdot \Delta_{\mathrm{c}} u^{\prime \prime} / \mathrm{J}$ & 10645.39 & 10401.53 & 9461.01 & 10101.03 \\
$\Delta_{\mathrm{c}} u^{\circ}(\mathrm{cr}) /.\left(\mathrm{J} \cdot \mathrm{g}^{-1}\right)$ & -26892.7 & -26898.5 & -26886.1 & -26870.4 \\
\hline$-\Delta_{\mathrm{c}} u^{\circ}(\mathrm{cr}) /.\left(\mathrm{J} \cdot \mathrm{g}^{-1}\right)$ & \multicolumn{5}{c}{$26886.9 \pm 6.1$} \\
\hline$\Delta_{\mathrm{c}} H_{\mathrm{m}}^{\circ}(\mathrm{cr})$ & $-4066.2 \pm 2.0$ \\
\hline
\end{tabular}

${ }^{a}$ The energy equivalent of the calorimeter: $\varepsilon_{\text {calor }}=(14816.3 \pm 1.0) \mathrm{J} \cdot \mathrm{K}^{-1}$. ${ }^{b}$ Uncertainties of the combustion energy are expressed as the standard deviation of the mean. 
Table S9. Compilation of Data on Molar Heat Capacities $C_{p, m}^{\circ}\left(\right.$ in $\left.\mathbf{J} \cdot \mathrm{K}^{-1} \cdot \mathbf{m o l}^{-1}\right)$ at $298.15 \mathrm{~K}$

\begin{tabular}{l|l|c|c|c}
\hline \multicolumn{1}{c|}{ Compounds } & \multicolumn{1}{c|}{$C_{\mathrm{p}, \mathrm{m}}^{\circ}(\mathrm{cr})^{a}$} & $-\Delta_{\mathrm{cr}}^{\mathrm{g}} C_{\mathrm{p}, \mathrm{m}}^{\circ}$ & $C_{\mathrm{p}, \mathrm{m}}^{\circ}(\mathrm{liq})^{a}$ & $-\Delta_{1}^{\mathrm{g}} C_{\mathrm{p}, \mathrm{m}}^{\circ}{ }^{b}$ \\
\hline 2- $\mathrm{CH}_{3}$-benzamide & 181.1 & 27.9 & 274.6 & 82.0 \\
3- $\mathrm{CH}_{3}$-benzamide & 181.1 & 27.9 & 274.6 & 82.0 \\
4- $\mathrm{CH}_{3}$-benzamide & 181.1 & 27.9 & 274.6 & 82.0 \\
2- $\mathrm{CH}_{3} \mathrm{O}$-benzamide & 230.9 & 35.4 & 304.4 & 89.7 \\
3- $\mathrm{CH}_{3} \mathrm{O}$-benzamide & 230.9 & 35.4 & 304.4 & 89.7 \\
4- $\mathrm{CH}_{3} \mathrm{O}$-benzamide & 230.9 & 35.4 & 304.4 & 89.7 \\
\hline
\end{tabular}

${ }^{a}$ Calculated according to the procedure developed by Chickos et al. $^{3}$ but some increments were adjusted for benzamide derivatives as follows.

Heat capacity in the crystal state: the increment for the amide group $\mathrm{CONH}_{2}=57.5 \mathrm{~J} \cdot \mathrm{K}^{-1} \cdot \mathrm{mol}^{-1}$ was adjusted specifically for benzamide derivatives using the experimental data ${ }^{5}$ for benzamide, $C_{\mathrm{p}, \mathrm{m}}^{\circ}(\mathrm{cr}$, benzamide $)=153.5 \mathrm{~J} \cdot \mathrm{K}^{-1} \cdot \mathrm{mol}^{-1}$.

Heat capacity in the liquid state: the increment for the amide group $\mathrm{CONH}_{2}=57.5 \mathrm{~J} \cdot \mathrm{K}^{-1} \cdot \mathrm{mol}^{-1}$ was adjusted specifically for benzamide derivatives using the experimental data ${ }^{5}$ for benzamide, $C_{\mathrm{p}, \mathrm{m}}^{\circ}($ liq, benzamide $)=246.2 \mathrm{~J} \cdot \mathrm{K}^{-1} \cdot \mathrm{mol}^{-1}$.

${ }^{\mathrm{b}}$ Calculated according to the procedure developed by Chickos and Acree. ${ }^{6}$

Temperature Adjustment of Fusion Enthalpies. Generally, the experimental molar enthalpy of fusion $\Delta_{\mathrm{cr}}^{1} H_{m}$ is referred to the melting temperature $T_{\text {fus }}$. The measured enthalpy of fusion have to be adjusted to the reference temperature $T=298 \mathrm{~K}$. The adjustment was calculated from the equation: ${ }^{6}$

$$
\left.\Delta_{\mathrm{cr}}^{1} H_{m}^{\circ}(298 \mathrm{~K})\right\} /\left(\mathrm{J} \cdot \mathrm{mol}^{-1}\right)=\Delta_{\mathrm{cr}}^{1} H_{m}^{\circ}\left(T_{\text {fus }} / \mathrm{K}\right)-\left(\Delta_{\mathrm{cr}}^{\mathrm{g}} C_{p, m}^{\circ}-\Delta_{1}^{\mathrm{g}} C_{p, m}^{\circ}\right) \times\left[\left(T_{\text {fus }} / \mathrm{K}\right)-298 \mathrm{~K}\right]
$$

where $\Delta_{\mathrm{cr}}^{\mathrm{g}} C_{p, m}^{\circ}$ and $\Delta_{1}^{\mathrm{g}} C_{p, m}^{\circ}$ are the differences of isobaric molar heat capacities (given in Table S9). With this adjustment the molar enthalpies of fusion, $\Delta_{\mathrm{cr}}^{1} H_{m}^{\circ}(298 \mathrm{~K})$, were calculated (see Table 4). 
Table S10. Compilation of Vapor Pressures Used for the Joint Treatment According to Eq 3 2- $\mathrm{CH}_{3}$-benzamide (ref. ${ }^{1}$ )

\begin{tabular}{|c|c|c|c|c|c|}
\hline$T / \mathrm{K}$ & $p / \mathrm{Pa}$ & $T / \mathrm{K}$ & $p / \mathrm{Pa}$ & $T / \mathrm{K}$ & $p / \mathrm{Pa}$ \\
\hline 325.1 & 0.08 & 325.1 & 0.08 & 325.1 & 0.08 \\
\hline 327.2 & 0.10 & 327.2 & 0.10 & 327.2 & 0.10 \\
\hline 329.2 & 0.13 & 329.2 & 0.13 & 329.2 & 0.13 \\
\hline 331.1 & 0.16 & 331.1 & 0.16 & 331.1 & 0.16 \\
\hline 333.2 & 0.21 & 333.2 & 0.21 & 333.2 & 0.20 \\
\hline 335.2 & 0.26 & 335.2 & 0.25 & 337.1 & 0.32 \\
\hline 337.1 & 0.32 & 337.1 & 0.32 & 339.2 & 0.40 \\
\hline 339.2 & 0.41 & 339.2 & 0.41 & 341.2 & 0.49 \\
\hline 341.2 & 0.51 & 341.2 & 0.50 & 343.1 & 0.62 \\
\hline 343.1 & 0.63 & 343.1 & 0.63 & 345.2 & 0.75 \\
\hline 347.2 & 0.96 & 345.2 & 0.78 & 347.2 & 0.93 \\
\hline \multicolumn{6}{|c|}{ 2- $\mathrm{CH}_{3}$-benzamide (this work) } \\
\hline$T / \mathrm{K}$ & $p / \mathrm{Pa}$ & $T / \mathrm{K}$ & $p / \mathrm{Pa}$ & $T / \mathrm{K}$ & $p / \mathrm{Pa}$ \\
\hline 329.4 & 0.13 & 350.2 & 1.31 & 362.2 & 4.35 \\
\hline 335.3 & 0.26 & 350.4 & 1.33 & 363.2 & 4.82 \\
\hline 338.1 & 0.37 & 351.2 & 1.44 & 365.0 & 5.73 \\
\hline 339.1 & 0.40 & 353.2 & 1.76 & 367.2 & 7.29 \\
\hline 341.2 & 0.51 & 353.3 & 1.80 & 367.3 & 7.07 \\
\hline 343.0 & 0.62 & 353.4 & 1.86 & 368.1 & 7.53 \\
\hline 343.2 & 0.65 & 354.4 & 2.07 & 368.2 & 7.52 \\
\hline 345.4 & 0.78 & 355.2 & 2.20 & 368.3 & 7.90 \\
\hline 346.0 & 0.87 & 356.1 & 2.44 & 369.3 & 8.69 \\
\hline 347.2 & 0.95 & 358.2 & 2.92 & 370.2 & 9.37 \\
\hline 347.8 & 1.05 & 358.2 & 2.84 & 371.2 & 10.00 \\
\hline 348.5 & 1.13 & 360.4 & 3.74 & 371.3 & 10.33 \\
\hline 349.3 & 1.25 & 361.2 & 3.99 & & \\
\hline 349.5 & 1.22 & 350.2 & 1.31 & & \\
\hline \multicolumn{6}{|c|}{ 3- $\mathrm{CH}_{3}$-benzamide (ref. ${ }^{1}$ ) } \\
\hline$T / \mathrm{K}$ & $p / \mathrm{Pa}$ & $T / \mathrm{K}$ & $p / \mathrm{Pa}$ & $T / \mathrm{K}$ & $p / \mathrm{Pa}$ \\
\hline 325.1 & 0.09 & 325.1 & 0.09 & 325.1 & 0.09 \\
\hline 327.2 & 0.12 & 327.2 & 0.12 & 327.2 & 0.12 \\
\hline 329.2 & 0.15 & 329.2 & 0.15 & 329.2 & 0.15 \\
\hline 331.1 & 0.19 & 331.1 & 0.19 & 331.1 & 0.19 \\
\hline 333.2 & 0.24 & 333.2 & 0.24 & 333.2 & 0.24 \\
\hline 337.1 & 0.38 & 335.2 & 0.30 & 335.2 & 0.30 \\
\hline 339.2 & 0.48 & 337.1 & 0.38 & 337.1 & 0.38 \\
\hline 343.1 & 0.75 & 339.2 & 0.48 & 339.2 & 0.47 \\
\hline 345.2 & 0.94 & 341.2 & 0.59 & 341.2 & 0.59 \\
\hline 347.2 & 1.17 & 345.2 & 0.92 & 343.1 & 0.74 \\
\hline \multicolumn{6}{|c|}{ 3- $\mathrm{CH}_{3}$-benzamide (this work) } \\
\hline$T / \mathrm{K}$ & $p / \mathrm{Pa}$ & $T / \mathrm{K}$ & $p / \mathrm{Pa}$ & $T / \mathrm{K}$ & $p / \mathrm{Pa}$ \\
\hline 329.7 & 0.16 & 349.5 & 1.42 & 360.4 & 4.14 \\
\hline 336.9 & 0.37 & 350.4 & 1.49 & 361.2 & 4.53 \\
\hline 338.1 & 0.41 & 351.0 & 1.66 & 362.5 & 5.18 \\
\hline 339.1 & 0.45 & 351.2 & 1.67 & 363.2 & 5.37 \\
\hline 341.2 & 0.56 & 353.2 & 2.00 & 364.8 & 6.19 \\
\hline 342.4 & 0.64 & 353.4 & 2.06 & 367.0 & 7.73 \\
\hline
\end{tabular}




\begin{tabular}{|c|c|c|c|c|c|}
\hline 343.0 & 0.69 & 353.7 & 2.05 & 367.2 & 7.87 \\
\hline 343.9 & 0.77 & 354.4 & 2.31 & 367.2 & 8.08 \\
\hline 345.4 & 0.88 & 355.2 & 2.46 & 367.3 & 7.83 \\
\hline 346.0 & 0.95 & 356.1 & 2.80 & 368.1 & 8.56 \\
\hline 346.3 & 0.98 & 356.3 & 2.81 & 369.3 & 9.65 \\
\hline 347.2 & 1.06 & 357.8 & 3.17 & 370.2 & 10.36 \\
\hline 348.5 & 1.25 & 360.0 & 3.96 & 371.2 & 11.02 \\
\hline \multicolumn{6}{|c|}{ 4- $\mathrm{CH}_{3}$-benzamide (ref. ${ }^{1}$ ) } \\
\hline$T / \mathrm{K}$ & $p / \mathrm{Pa}$ & $T / \mathrm{K}$ & $p / \mathrm{Pa}$ & $T / \mathrm{K}$ & $p / \mathrm{Pa}$ \\
\hline 339.1 & 0.12 & 339.1 & 0.11 & 339.1 & 0.12 \\
\hline 341.2 & 0.15 & 341.2 & 0.15 & 341.2 & 0.14 \\
\hline 343.2 & 0.18 & 343.2 & 0.18 & 343.2 & 0.18 \\
\hline 345.1 & 0.23 & 345.1 & 0.22 & 347.2 & 0.28 \\
\hline 349.2 & 0.35 & 349.2 & 0.35 & 349.2 & 0.35 \\
\hline 351.1 & 0.43 & 351.1 & 0.43 & 351.1 & 0.42 \\
\hline 353.2 & 0.54 & 353.2 & 0.53 & 353.2 & 0.52 \\
\hline 355.2 & 0.65 & 355.2 & 0.65 & 355.2 & 0.63 \\
\hline 357.1 & 0.80 & 357.1 & 0.81 & 357.1 & 0.79 \\
\hline 359.1 & 0.99 & 359.1 & 1.00 & 359.1 & 0.97 \\
\hline 361.2 & 1.20 & 361.2 & 1.18 & 361.2 & 1.17 \\
\hline \multicolumn{6}{|c|}{ 4- $\mathrm{CH}_{3}$-benzamide (this work) } \\
\hline$T / \mathrm{K}$ & $p / \mathrm{Pa}$ & $T / \mathrm{K}$ & $p / \mathrm{Pa}$ & $T / \mathrm{K}$ & $p / \mathrm{Pa}$ \\
\hline 343.9 & 0.20 & 359.5 & 1.00 & 367.5 & 2.22 \\
\hline 346.6 & 0.26 & 361.3 & 1.21 & 367.7 & 2.29 \\
\hline 350.4 & 0.40 & 361.8 & 1.28 & 368.7 & 2.49 \\
\hline 352.7 & 0.51 & 362.5 & 1.37 & 369.5 & 2.65 \\
\hline 354.6 & 0.62 & 363.6 & 1.54 & 371.5 & 3.17 \\
\hline 355.9 & 0.71 & 364.6 & 1.65 & & \\
\hline 356.0 & 0.71 & 365.7 & 1.85 & & \\
\hline \multicolumn{6}{|c|}{ 2- $\mathrm{CH}_{3} \mathrm{O}$-benzamide (Ref. ${ }^{2}$ ) } \\
\hline$T / \mathrm{K}$ & $p / \mathrm{Pa}$ & $T / \mathrm{K}$ & $p / \mathrm{Pa}$ & $T / \mathrm{K}$ & $p / \mathrm{Pa}$ \\
\hline 335.1 & 0.10 & 335.1 & 0.10 & 335.1 & 0.10 \\
\hline 337.2 & 0.13 & 337.2 & 0.13 & 337.2 & 0.12 \\
\hline 339.2 & 0.16 & 339.2 & 0.16 & 339.2 & 0.15 \\
\hline 341.1 & 0.20 & 341.1 & 0.20 & 341.1 & 0.20 \\
\hline 343.2 & 0.25 & 343.2 & 0.25 & 343.2 & 0.24 \\
\hline 345.2 & 0.31 & 345.2 & 0.31 & 345.2 & 0.30 \\
\hline 347.1 & 0.39 & 347.1 & 0.39 & 347.1 & 0.38 \\
\hline 349.2 & 0.48 & 349.2 & 0.49 & 349.2 & 0.47 \\
\hline 351.2 & 0.59 & 351.2 & 0.59 & 351.2 & 0.58 \\
\hline 353.1 & 0.74 & 353.1 & 0.75 & 353.1 & 0.74 \\
\hline 355.2 & 0.92 & 355.2 & 0.93 & 355.2 & 0.91 \\
\hline 357.2 & 1.12 & 357.2 & 1.12 & 357.2 & 1.11 \\
\hline \multicolumn{6}{|c|}{ 2- $\mathrm{CH}_{3} \mathrm{O}$-benzamide (this work) } \\
\hline$T / \mathrm{K}$ & $p / \mathrm{Pa}$ & $T / \mathrm{K}$ & $p / \mathrm{Pa}$ & $T / \mathrm{K}$ & $p / \mathrm{Pa}$ \\
\hline 368.4 & 3.20 & 381.4 & 10.41 & 396.2 & 36.69 \\
\hline 370.4 & 3.88 & 384.2 & 13.39 & 398.5 & 45.94 \\
\hline 373.2 & 4.98 & 388.6 & 19.47 & & \\
\hline 378.8 & 8.24 & 394.2 & 31.69 & & \\
\hline
\end{tabular}

3- $\mathrm{CH}_{3} \mathrm{O}$-benzamide (Ref. $\left.{ }^{2}\right)$ 


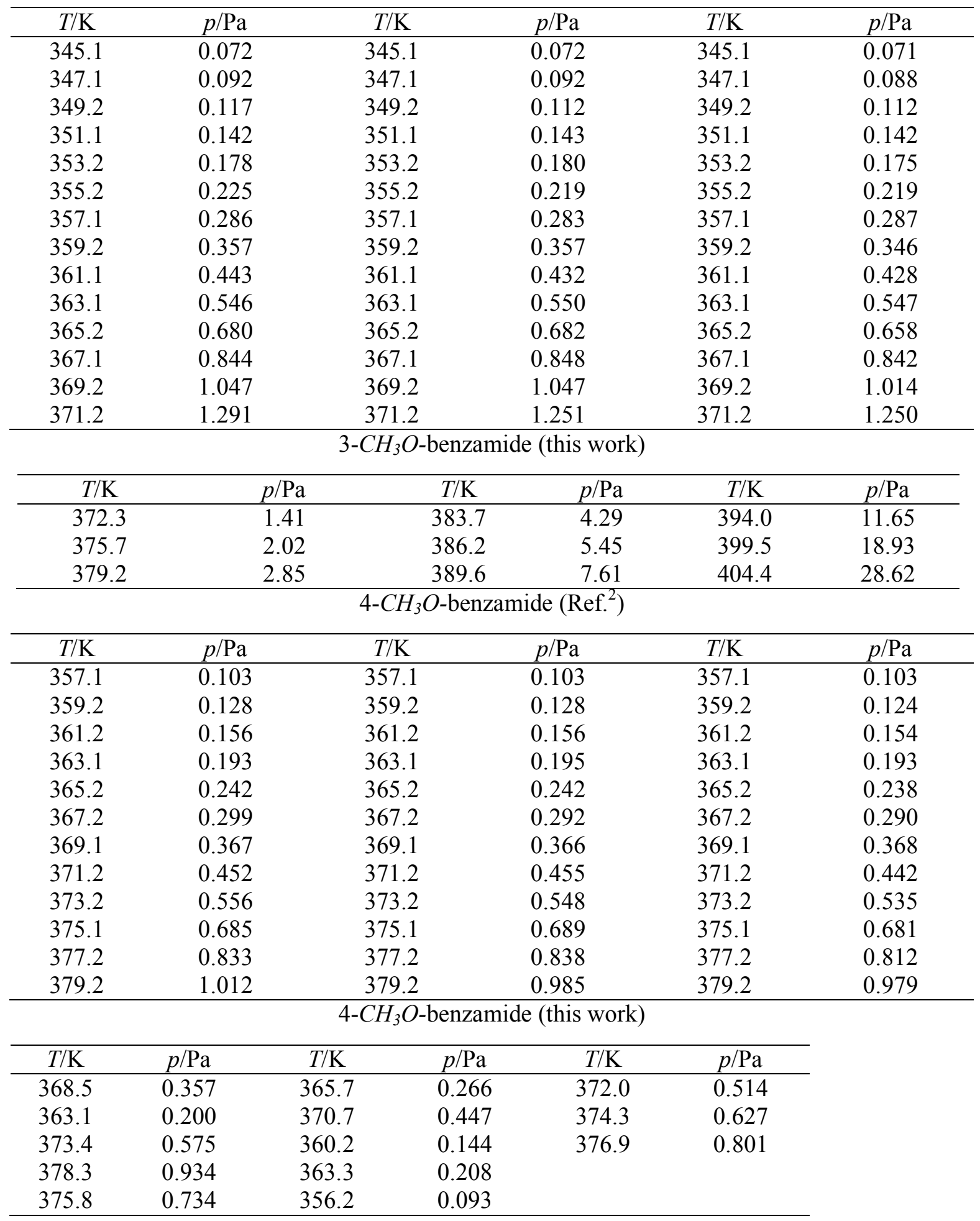


Table S 11. Coefficients of Eq. 3 for Calculation of Vapor Pressures over Solid Benzamides

\begin{tabular}{l|l|c|c|}
\hline \multicolumn{1}{c|}{ Compounds } & $\Delta_{\mathrm{cr}}^{\mathrm{g}} G_{m}^{\circ}(298 \mathrm{~K})$ & $\Delta_{\mathrm{cr}}^{\mathrm{g}} H_{m}^{\circ}(298 \mathrm{~K})$ & $-\Delta_{\mathrm{cr}}^{\mathrm{g}} C_{\mathrm{p}, \mathrm{m}}^{\circ} a$ \\
\hline & \multicolumn{1}{c|}{$\mathrm{kJ} \cdot \mathrm{mol}^{-1}$} & $\mathrm{~kJ} \cdot \mathrm{mol}^{-1}$ & $\mathrm{~J} \cdot \mathrm{K}^{-1} \cdot \mathrm{mol}^{-1}$ \\
\hline 2-methylbenzamide & $43.7 \pm 0.6$ & $107.2 \pm 0.5$ & 27.9 \\
3-methylbenzamide & $43.2 \pm 0.6$ & $106.0 \pm 0.5$ & 27.9 \\
4-methylbenzamide & $47.1 \pm 0.7$ & $109.7 \pm 0.6$ & 27.9 \\
2-methoxybenzamide & $46.2 \pm 0.8$ & $109.2 \pm 0.7$ & 35.4 \\
3-methoxybenzamide & $51.4 \pm 0.7$ & $120.3 \pm 0.6$ & 35.4 \\
4-methoxybenzamide & $53.8 \pm 0.7$ & $119.7 \pm 0.6$ & 35.4 \\
\hline
\end{tabular}

${ }^{a}$ From Table S3.

Table S12. Enthalpies of Vaporization, $\Delta_{1}^{\mathrm{g}} H_{m}^{\circ}$, of Benzamides (in kJ·mol ${ }^{-1}$ )

\begin{tabular}{|c|c|c|c|c|c|}
\hline Compounds & $\mathrm{M}^{a}$ & $T$-range & $\begin{array}{c}\Delta_{1}^{\mathrm{g}} H_{m}^{\circ} / \\
T_{\mathrm{av}}\end{array}$ & $\begin{array}{l}\Delta_{1}^{\mathrm{g}} H_{m}^{\circ} / \\
298 \mathrm{~K}^{b}\end{array}$ & Ref \\
\hline benzamide (liq) & $\mathrm{S}$ & $380.4-438.2$ & $77.3 \pm 1.3$ & $85.4 \pm 1.5$ & 7 \\
\hline \multirow{2}{*}{ 2- $\mathrm{CH}_{3}$-benzamide (liq) } & & 298 & & $90.7 \pm 1.5$ & \multirow{2}{*}{$\begin{array}{l}\text { from Table } 4 \\
\text { additive }\end{array}$} \\
\hline & & 298 & & $89.6 \pm 1.5$ & \\
\hline \multirow{2}{*}{ 3- $\mathrm{CH}_{3}$-benzamide (liq) } & & 298 & & $89.5 \pm 0.9$ & \multirow{2}{*}{$\begin{array}{l}\text { from Table } 4 \\
\text { additive }\end{array}$} \\
\hline & & 298 & & $89.6 \pm 1.5$ & \\
\hline \multirow[t]{2}{*}{ 4- $\mathrm{CH}_{3}$-benzamide (liq) } & & 298 & & $91.4 \pm 1.7$ & \multirow{2}{*}{$\begin{array}{l}\text { from Table } 4 \\
\text { additive }\end{array}$} \\
\hline & & 298 & & $89.6 \pm 1.5$ & \\
\hline \multirow[t]{3}{*}{ 2- $\mathrm{CH}_{3} \mathrm{O}$-benzamide (liq) } & $\mathrm{T}$ & $402.3-425.7$ & $79.1 \pm 0.7$ & $89.4 \pm 1.1$ & \multirow{3}{*}{$\begin{array}{l}\text { this work } \\
\text { from Table } 4 \\
\text { additive }\end{array}$} \\
\hline & & 298 & & $89.4 \pm 1.8$ & \\
\hline & & & & $97.9 \pm 1.5$ & \\
\hline \multirow[t]{3}{*}{ 3- $\mathrm{CH}_{3} \mathrm{O}$-benzamide (liq) } & $\mathrm{T}$ & $408.2-432.3$ & $87.4 \pm 1.0$ & $98.3 \pm 1.3$ & \multirow{3}{*}{$\begin{array}{l}\text { this work } \\
\text { from Table } 4 \\
\text { additive }\end{array}$} \\
\hline & & 298 & & $97.5 \pm 1.9$ & \\
\hline & & 298 & & $97.9 \pm 1.5$ & \\
\hline \multirow[t]{2}{*}{ 4- $\mathrm{CH}_{3} \mathrm{O}$-benzamide (liq) } & & 298 & & $97.6 \pm 2.4$ & \multirow{2}{*}{$\begin{array}{l}\text { from Table } 4 \\
\text { additive }\end{array}$} \\
\hline & & 298 & & $97.9 \pm 1.5$ & \\
\hline
\end{tabular}

${ }^{a}$ Methods: $\mathrm{S}=$ static method; $\mathrm{T}=$ transpiration method. ${ }^{b}$ Uncertainties of sublimation enthalpies are expressed in this table as standard deviations. Recommended values are given in bold.

Table S13. The G4 Total Energies at $0 \mathrm{~K}$ and Enthalpies at 298.15 $\mathrm{K}$ (in Hartree) of the Molecules Studied in This Work

\begin{tabular}{lcc}
\hline \multirow{2}{*}{\multicolumn{1}{c}{ Compounds }} & \multicolumn{2}{c}{$\mathrm{G} 4$} \\
\cline { 2 - 3 } & $\mathrm{E}_{0}$ & $\mathrm{H}_{298}$ \\
\hline 2- $\mathrm{CH}_{3}$-benzamide & -440.024166 & -440.014025 \\
3- $\mathrm{CH}_{3}$-benzamide & -440.026337 & -440.015916 \\
4- $\mathrm{CH}_{3}$-benzamide & -440.026475 & -440.016029 \\
2- $\mathrm{CH}_{3} \mathrm{O}$-benzamide & -515.212121 & -515.200915 \\
3- $\mathrm{CH}_{3} \mathrm{O}$-benzamide & -515.211992 & -515.200868 \\
4- $\mathrm{CH}_{3} \mathrm{O}$-benzamide & -515.211971 & -515.200860 \\
benzene & -232.093987 & -232.088586 \\
toluene & -271.377414 & -271.370151 \\
benzamide & -400.742537 & -400.734009 \\
\hline
\end{tabular}


Table S14 Thermochemical data at $T=298 \mathrm{~K}\left(p^{\circ}=0.1 \mathrm{MPa}\right)$ for reference compounds (in $\mathrm{kJ} \cdot \mathrm{mol}^{-1}$ )

\begin{tabular}{llcc}
\hline & \multicolumn{1}{c}{$\Delta_{\mathrm{f}} H_{m}^{\circ}(\mathrm{liq})$} & \multicolumn{1}{c}{$\Delta_{\mathrm{l}}^{\mathrm{g}} H_{m}^{\circ}$} & $\Delta_{\mathrm{f}} H_{m}^{\circ}(\mathrm{g})_{\exp .}$ \\
\hline benzene & $49.0 \pm 0.9^{8}$ & $33.9 \pm 0.1^{8}$ & $82.9 \pm 0.9$ \\
methyl-benzene & $12.0 \pm 1.1^{8}$ & $38.1 \pm 0.1^{8}$ & $50.1 \pm 1.1$ \\
methoxy-benzene & $-117.1 \pm 1.4^{9}$ & $46.4 \pm 0.3^{9}$ & $-70.7 \pm 1.4$ \\
phenol & $-154.2 \pm 0.8^{10}$ & $57.8 \pm 0.5^{10}$ & $-96.4 \pm 0.9$ \\
benzamide & $-202.5 \pm 0.5^{5}$ & $102.7 \pm 0.4^{7}$ & $-99.8 \pm 0.6$ \\
\hline
\end{tabular}

Table S15. Comparison of Experimental and G4-Calculated Enthalpies of Formation of Benzamides $\left(\mathbf{k J} \cdot \mathbf{m o l}^{-1}\right)$

\begin{tabular}{lccc}
\hline \multicolumn{1}{c}{ Compounds } & $\begin{array}{c}\Delta_{\mathrm{f}} H_{m}^{\circ}(\mathrm{g}) \\
\exp \end{array}$ & $\begin{array}{c}\Delta_{\mathrm{f}} H_{m}^{\circ}(\mathrm{g}) \\
\text { AT }\end{array}$ & $\begin{array}{c}\Delta_{\mathrm{f}} H_{m}^{\circ}(\mathrm{g}) \\
\text { eq 12 }\end{array}$ \\
\hline 2- $\mathrm{CH}_{3}$-benzamide & $-128.7 \pm 1.7$ & -128.1 & -127.8 \\
3- $\mathrm{CH}_{3}$-benzamide & $-132.8 \pm 1.6$ & -133.0 & -132.8 \\
4- $\mathrm{CH}_{3}$-benzamide & $-136.7 \pm 1.5$ & -133.4 & -133.1 \\
2- $\mathrm{CH}_{3} \mathrm{O}$-benzamide & $-255.5 \pm 1.5$ & -257.0 & -256.6 \\
3- $\mathrm{CH}_{3} \mathrm{O}$-benzamide & $-255.3 \pm 1.7$ & -256.9 & -256.5 \\
4- $\mathrm{CH}_{3} \mathrm{O}$-benzamide & $-257.4 \pm 1.8$ & -256.9 & -256.5 \\
\hline
\end{tabular}




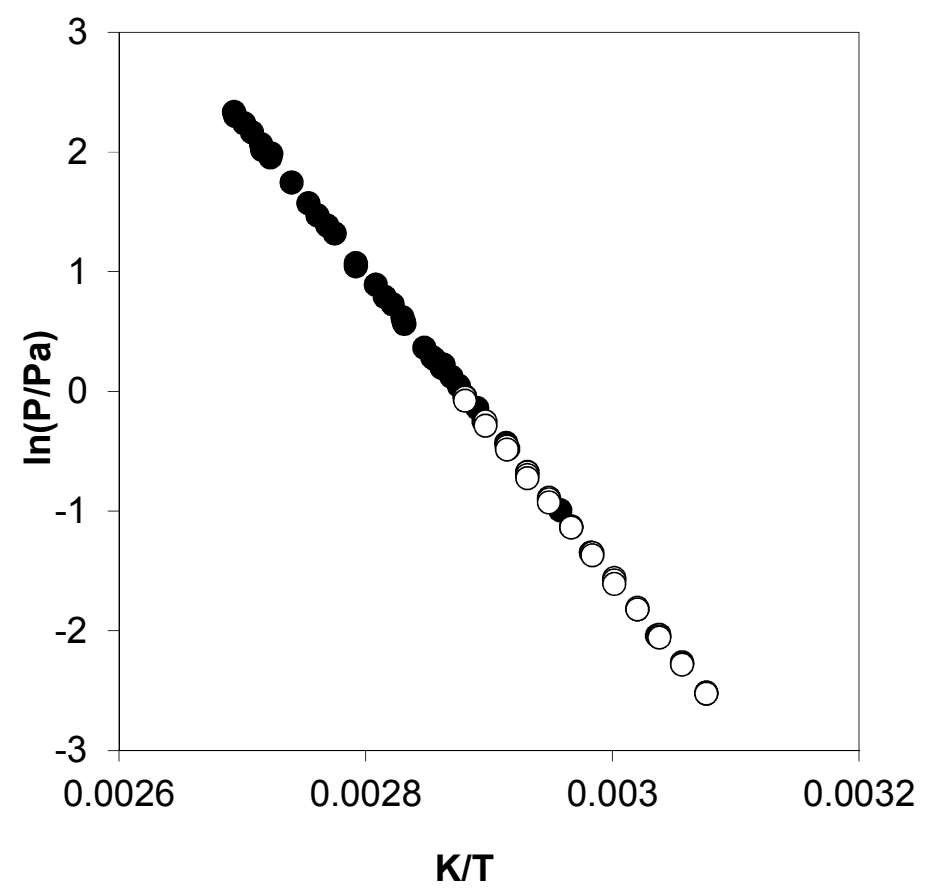

Figure S1. Experimental vapor pressures of the 2- $\mathrm{CH}_{3}$-benzamide: - - from this work and o- from ref. ${ }^{1}$

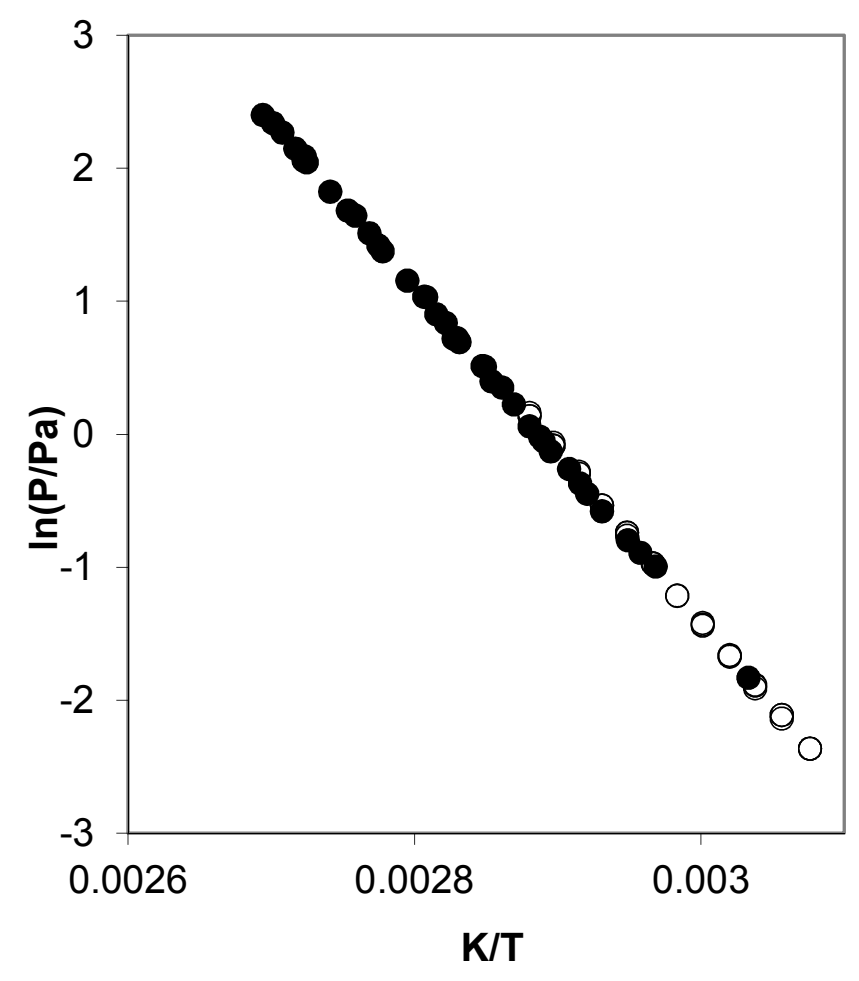

Figure S2. Experimental vapor pressures of the 3- $\mathrm{CH}_{3}$-benzamide: - - from this work and o- from ref. ${ }^{1}$ 


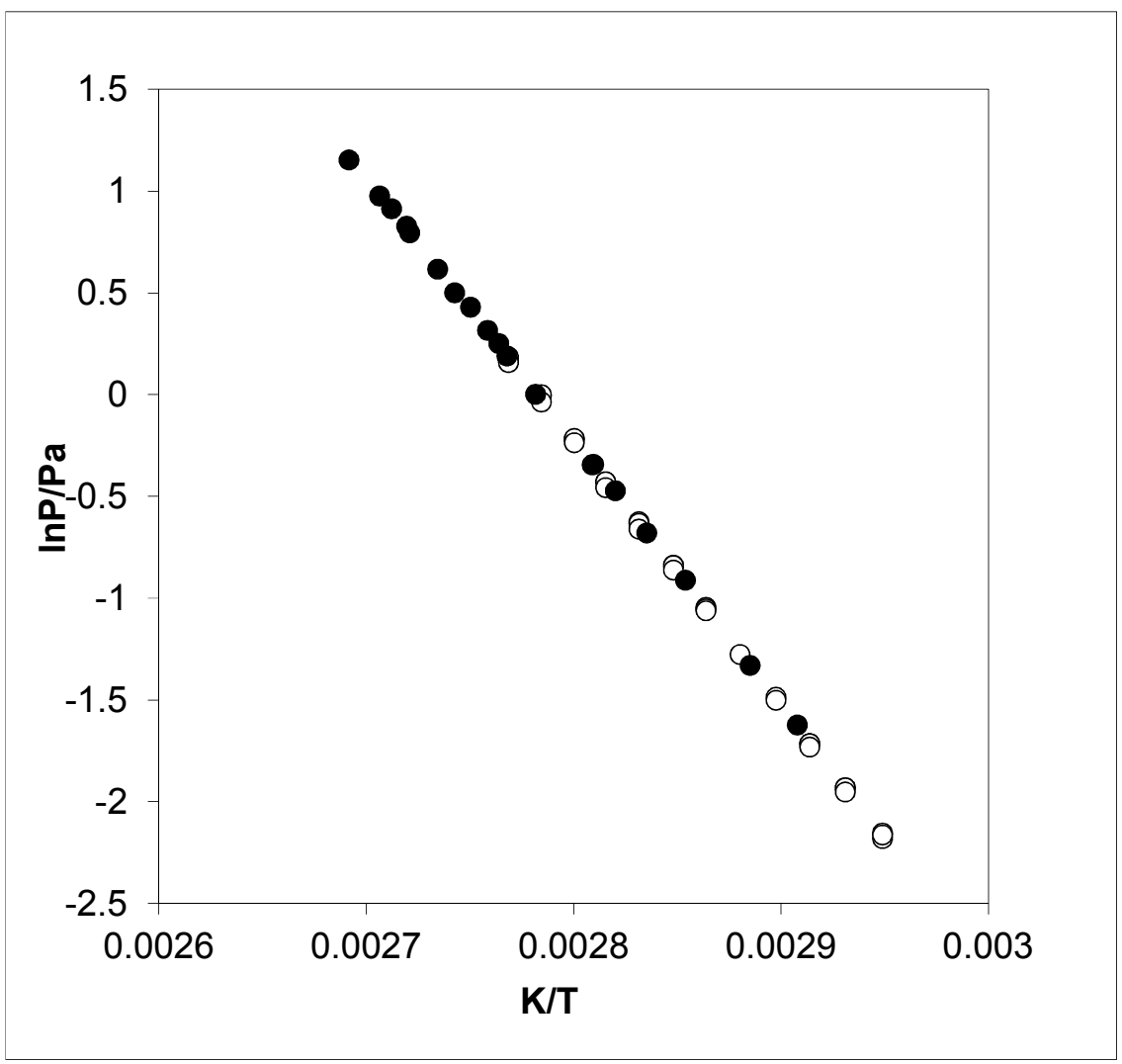

Figure S3. Experimental vapor pressures of the 4- $\mathrm{CH}_{3}$-benzamide: - - from this work and o- from ref. ${ }^{1}$

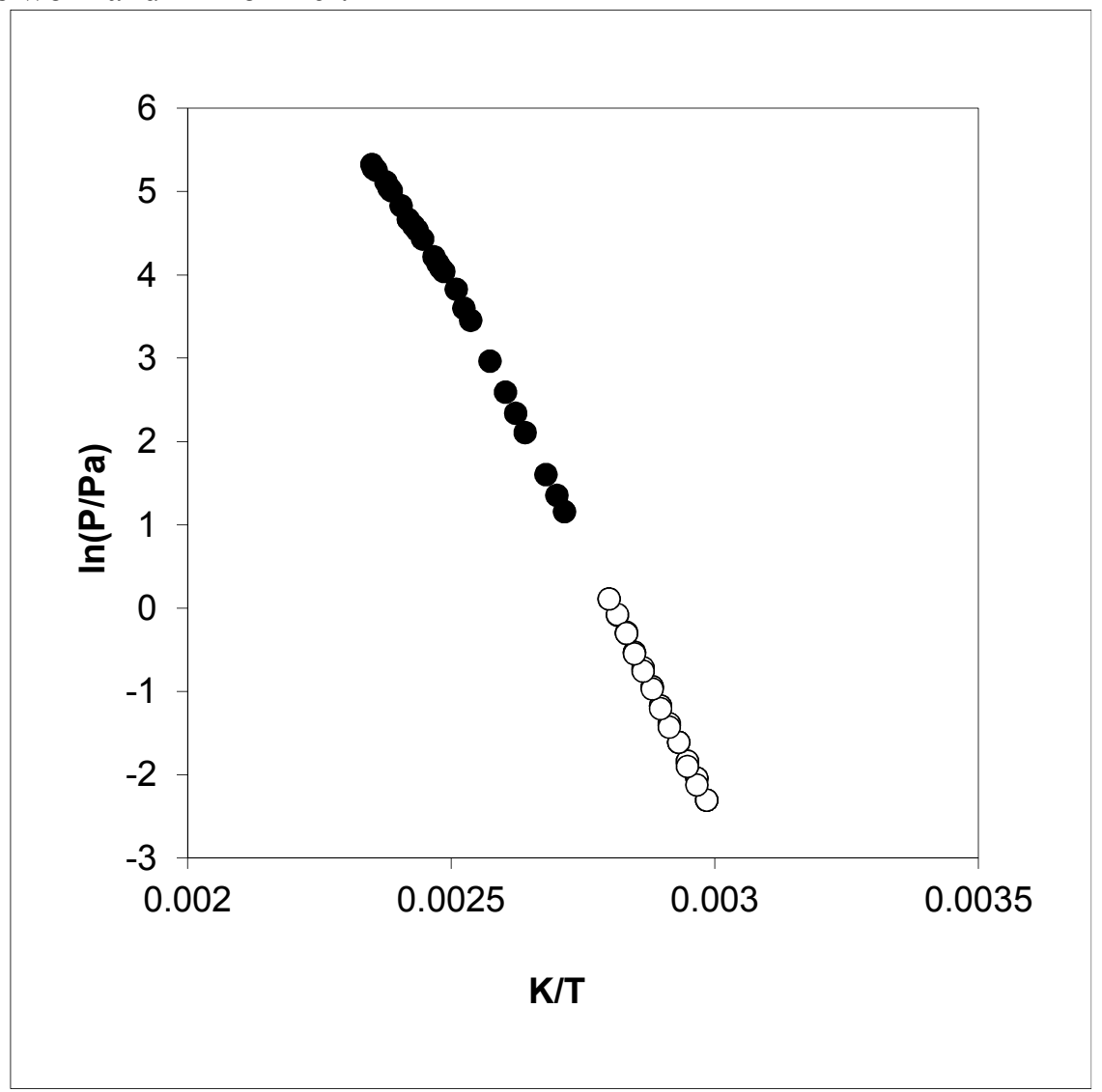

Figure S4. Experimental vapor pressures of the 2- $\mathrm{CH}_{3} \mathrm{O}$-benzamide:

- - from this work and ०- from ref. ${ }^{2}$ 


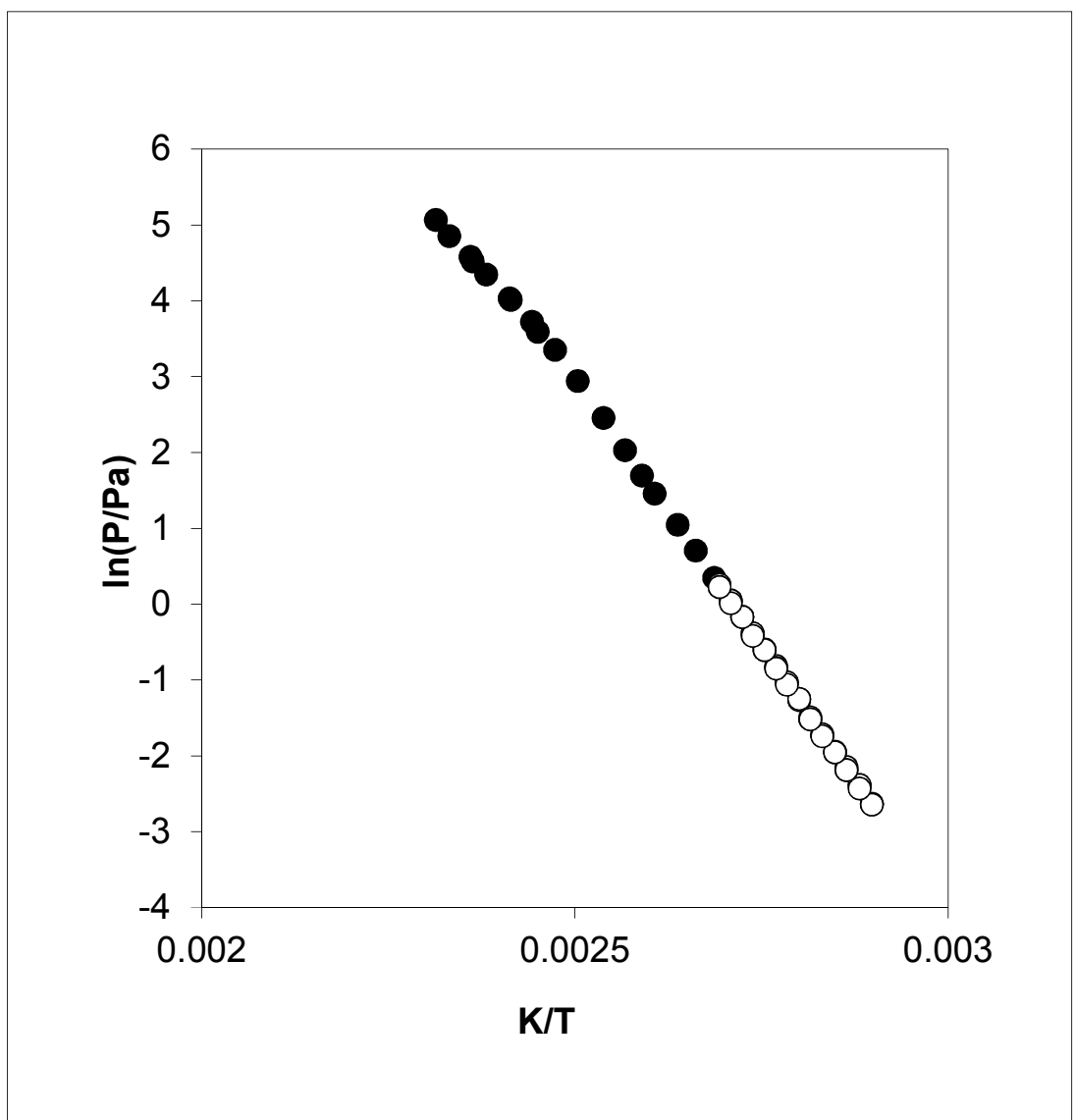

Figure S5. Experimental vapor pressures of the 3- $\mathrm{CH}_{3} \mathrm{O}$-benzamide: - - from this work and o- from ref. ${ }^{2}$

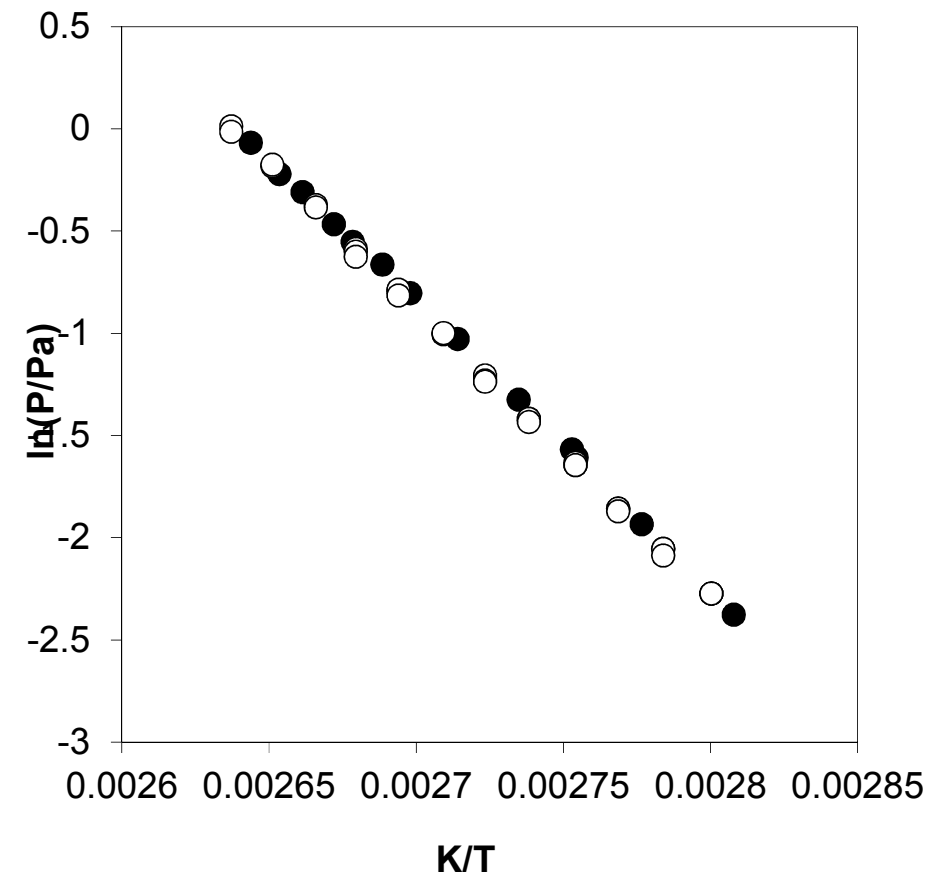

Figure S6. Experimental vapor pressures of the 4- $\mathrm{CH}_{3} \mathrm{O}$-benzamide:

- - from this work and o- from ref. ${ }^{2}$ 


\section{REFERENCES}

(1) Almeida, A. R. R. P.; Matos, M. A. R.; Monte M. J. S.; Morais, V. M. F. Experimental and Computational Thermodynamic Study of ortho-, meta-, and para-Methylbenzamide. J. Chem. Thermodyn. 2012, 47, 81-89.

(2) Almeida, A. R. R. P.; Monte, M. J. S.; Matos, M. A. R.; Morais, V. M. F. The Thermodynamic Stability of the Three Isomers of Methoxybenzamide: An Experimental and Computational Study. J. Chem. Thermodyn. 2014, 73, 12-22.

(3) Chickos, J. S.; Hosseini, S.; Hesse, D. G.; Liebman, J. F. Heat Capacity Corrections to a Standard State: a Comparison of New and Some Literature Methods for Organic Liquids and Solids. Struct. Chem. 1993, 4, 271-277.

(4) Hubbard, W. N.; Scott, D.W.; Waddington, G. In Experimental Thermochemistry, in: F.D. Rossini (Ed.), Interscience Publishers, New York, 1956, pp. 75-127.

(5) Steele, W.V.; Chirico, R.D.; Nguyen, A.; Hossenlopp, I.A.; Smith, N.K., Determination of Ideal-Gas Enthalpies of Formation for Key Compounds. Am. Inst. Chem. Eng. Symp. Ser. (AIChE Symp. Ser.) 1990, 138-154.

(6) Chickos, J. S.; Acree, W. E. Jr, Enthalpies of Sublimation of Organic and Organometallic Compounds. 1910-2001, J. Phys. Chem. Ref. Data 2002, 31, 537-698.

(7) Almeida, A. R. R. P.; Monte, M. J. S. Thermodynamic Study of Benzamide, NMethylbenzamide, and N,N-Dimethylbenzamide: Vapor Pressures, Phase Diagrams, and Hydrogen Bond Enthalpy. J. Chem. Eng. Data, 2010, 55, 3507-3512.

(8) Roux, M. V.; Temprado, M.; Chickos, J. S.; Nagano, Y. Critically Evaluated Thermochemical Properties of Polycyclic Aromatic Hydrocarbons. J. Phys. Chem. Ref. Data 2008, 37, 1855-1996.

(9) Simões, R. G.; Agapito, F.; Diogo, H. P.; Minas da Piedade, M. E. The Enthalpy of Formation of Anisole: Implications for the Controversy on the $\mathrm{O}-\mathrm{H}$ Bond Dissociation Enthalpy in Phenol. J. Phys. Chem. A 2014, 118, 11026-11032.

(10) Andon, R.J.L.; Biddiscombe, D.P.; Cox, J.D.; Handley, R.; Harrop, D.; Herington, E.F.G.; Martin, J.F., Thermodynamic properties of organic oxygen compounds. Part I. Preparation and Physical Properties of Pure Phenol, Cresols, and Xylenols. J. Chem. Soc. 1960, 5246-5254. 